\title{
Aberrant Gating of Photic Input to the Suprachiasmatic Circadian Pacemaker of Mice Lacking the $\mathrm{VPAC}_{2}$ Receptor
}

\author{
Alun T. Hughes, Briana Fahey, David J. Cutler, Andrew N. Coogan, and Hugh D. Piggins \\ School of Biological Sciences, University of Manchester, Manchester M13 9PT, United Kingdom
}

VIP acting via the $\mathrm{VPAC}_{2}$ receptor is implicated as a key signaling pathway in the maintenance and resetting of the hypothalamic suprachiasmatic nuclei (SCN) circadian pacemaker; circadian rhythms in SCN clock gene expression and wheel-running behavior are abolished in mice lacking the $\mathrm{VPAC}_{2}$ receptor $\left(\mathrm{Vipr}^{-{ }^{-1}}\right.$ ). Here, using immunohistochemical detection of pERK (phosphorylated extracellular signal-regulated kinases 1/2) and c-FOS, we tested whether the gating of photic input to the SCN is maintained in these apparently arrhythmic Vipr $2^{-/-}$mice. Under light/dark and constant darkness, spontaneous expression of pERK and c-FOS in the wild-type mouse SCN was significantly elevated during subjective day compared with subjective night; no diurnal or circadian variation in pERK or c-FOS was detected in the SCN of $\mathrm{Vipr}^{-/-}$mice. In constant darkness, light pulses given during the subjective night but not the subjective day significantly increased expression of pERK and c-FOS in the wild-type SCN. In contrast, light pulses given during both subjective day and subjective night robustly increased expression of pERK and c-FOS in the Vipr2 ${ }^{-/}$mouse SCN. Although photic stimuli activate intracellular pathways within the $\mathrm{SCN}$ of $\mathrm{Vipr}^{-/-}$mice, they do not engage the core clock mechanisms. The absence of photic gating, together with the general lack of overt rhythms in circadian output, strongly suggests that the SCN circadian pacemaker is completely dysfunctional in the Vipr2 ${ }^{-/-}$mouse.

Key words: circadian; vasoactive intestinal polypeptide; mitogen-activated protein kinase; wheel running; entrainment; suprachiasmatic nuclei

\section{Introduction}

The suprachiasmatic nuclei of the hypothalamus (SCN) contain the dominant mammalian circadian pacemaker (Rusak and Zucker, 1979). Compelling evidence from the past decade has identified many of the canonical clock genes of this pacemaker and their roles in biological timekeeping (Allada et al., 2001; Reppert and Weaver, 2002). The SCN pacemaker is entrained to regularly recurring environmental signals (Zeitgebers) of which daily variation in lighting levels is among the most important.

Many intracellular cascades mediate the actions of light on the rodent SCN pacemaker (Gillette and Mitchell, 2002). Among these, the phosphorylation of extracellular signal-regulated kinases $1 / 2$ (ERK) and induction of the immediate early gene product c-FOS are robust markers of SCN neuronal activation by photic stimuli (Abe and Rusak, 1994; Obrietan et al., 1998; Coogan and Piggins, 2003). Large increases in phosphorylated ERK (pERK)-immunoreactivity (-ir) and c-FOS-ir are induced in the nocturnal rodent $\mathrm{SCN}$ in response to short-duration $(\sim 15 \mathrm{~min})$ light pulses given during the animals' active (subjective night) phase. In contrast, light pulses given during the animals' rest

Received Dec. 3, 2003; revised Feb. 23, 2004; accepted Feb. 28, 2004.

This work was supported by project grants from the Biotechnology and Biological Sciences Research Council (BBSRC) to H.D.P. and a BBSRC studentship to A.T.H. We thank Owen Jones and Rob Lucas for comments on a previous draft of this manuscript, Rayna Samuels for excellent technical assistance, and Tony Harmar for supplying the original breeding stock of Vipr2 ${ }^{-1-}$ mice.

Correspondence should be addressed to Dr. Hugh D. Piggins, 3.614 Stopford Building, School of Biological Sciences, University of Manchester, 0xford Road, Manchester M13 9PT, UK. E-mail: hugh.piggins@man.ac.uk. DOI:10.1523/JNEUROSCI.5345-03.2004

Copyright $\odot 2004$ Society for Neuroscience $\quad$ 0270-6474/04/243522-05\$15.00/0 (subjective day) phase fail to increase the expression of pERK-ir or c-FOS-ir in the rodent SCN. This "gating" of responses to photic inputs is a key property of the mammalian SCN pacemaker and presumably underlies the phase dependency of phase resetting of behavioral rhythms by light.

Retinorecipient cells of the rodent SCN include those that synthesize VIP. VIP and one of its receptors, $\mathrm{VPAC}_{2}$, are expressed in the SCN and have emerged as a critical neuropeptidesignaling pathway in both photic entrainment and maintenance of circadian phase (for review, see Piggins and Cutler, 2003). For example, mice carrying a null mutation to the $\mathrm{VPAC}_{2}$ receptor gene Vipr2 $2^{-/-}$do not manifest overt, coherent rhythms in canonical clock genes, SCN neuronal activity, or wheel-running behavior (Harmar et al., 2002; Cutler et al., 2003).

Surprisingly, in all mouse models in which the circadian pacemaker has been severely compromised, only the output of the pacemaker has been examined thoroughly. Here, using photic induction of pERK and c-FOS, we demonstrate a profound absence of gating of photic input to the SCN of Vipr $2^{-/-}$mice.

\section{Materials and Methods}

Animals. Male and female Vipr2 ${ }^{-1-}$ (eighth generation) (Cutler et al., 2003) and wild-type (WT) (C57BL/6J; University of Manchester breeding colony) mice of at least 6 weeks of age were used in this study. All animals were maintained under a $12 \mathrm{hr}$ light/dark (LD) cycle [where Zeitgeber time (ZT) 0 is the onset of the light phase; $\sim 60$ lux lights on] before the study with ad libitum access to food and water. All procedures were performed in accordance with the United Kingdom Animals (Scientific Procedures) Act 1986. 
Behavioral analysis. WT and Vipr $2^{-1-}$ mice were housed individually in running wheel-equipped cages. Animals were maintained under a LD cycle for $7-10 \mathrm{~d}$ ( $\sim 60$ lux lights on), followed by constant darkness (DD) for an additional $10 \mathrm{~d}$ to monitor circadian expression of wheel-running behavior, then returned to LD. Wheel revolutions were recorded in 10 min time bins, and $\chi^{2}$ periodogram analyses were performed (Tau software; Mini-Mitter, Sunriver, OR) to determine free-running activity periods and to analyze wheel-running activity patterns. Experienced observers blind to genotype also rated occurrence of rhythmic activity. In $88 \%$ of cases, visual inspection of actograms and periodogram analysis were in agreement; in the remaining $12 \%$, periodogram analysis was used to classify individuals as rhythmic or arrhythmic. In DD, circadian time (CT) 12 was defined as the onset of the main wheel-running activity period.

Light treatments and tissue collection. WT and Vipr $2^{-1-}$ mice were sampled across the diurnal cycle at $4 \mathrm{hr}$ intervals. For circadian expression and photic induction protocols, animals previously entrained under LD were sampled at $4 \mathrm{hr}$ intervals during the second cycle of DD (Aschoff type II protocol) either in DD or after a light pulse. CT12 was defined as a projection of lights off under the previous LD cycle. For photic induction protocols, animals were sampled immediately (pERK and ERK) or 1 hr (c-FOS) after a 15 min light pulse ( $\sim 60$ lux). Brains were removed after halothane anesthesia and cervical dislocation, fixed in $4 \%$ paraformaldehyde solution in $0.1 \mathrm{~m}$ phosphate buffer for $48-72 \mathrm{hr}$, then cryoprotected in $30 \%$ sucrose for $48-72 \mathrm{hr}$. Brains were quick frozen with dry ice, and $30 \mu \mathrm{m}$ coronal sections were cut through the extent of the SCN.

Immunohistochemistry. Immunoreactivity to pERK, ERK (total pERK and unphosphorylated ERK), and c-FOS was detected using standard techniques as described by Coogan and Piggins (2003). Briefly, sections were incubated with rabbit polyclonal antibodies raised against pERK (1:250; Cell Signaling Technologies, Hitchin, UK), ERK (1:150; Santa Cruz Biotechnology, Calne, UK), or c-FOS (1:8000; Santa Cruz Biotechnology) at $4^{\circ} \mathrm{C}$ for $48 \mathrm{hr}$, followed by biotinylated goat anti-rabbit (90 min; 1:400; Vector Laboratories, Peterborough, UK), then ABC solution (90 min; 1:400; Vectastain Elite ABC Kit, Vector Laboratories) before substrate visualization using nickel DAB (Sigma, Poole, UK).

Analysis. Images of pERK-ir and ERK-ir were captured using a microscope-mounted digital camera (Camedia C-2020Z; Olympus, London, UK) and processed in Adobe Photoshop Elements (Adobe Systems, San Jose, CA) and Scion Image for Windows (Scion, Frederick, MD). c-FOS-ir nuclei per SCN section were counted manually. Levels of immunoreactivity/numbers of nuclei were analyzed throughout the SCN and averaged to give a mean value per section for each animal. Statistical significance $(p<0.05)$ was determined using two-way and three-way ANOVAs with a priori pairwise comparisons (SYSTAT version 10; SPSS, Chicago, IL). Confidence intervals (CIs; 95\%) were used to assess how individual points compared with relevant group means.

\section{Results}

Behavioral phenotype of Vipr2 ${ }^{-/-}$mice

Mice homozygous for the disrupted Vipr2 allele showed apparent entrainment to a LD cycle with a very close association between the onset of activity and the onset of darkness (Fig. 1A,B). The majority of Vipr $2^{-/-}$mice did not display overt circadian rhythmicity in wheel-running behavior in DD ( $n=12$ of 18). Many bouts of activity ( $\sim 30-150$ min duration) were dispersed across the circadian cycle with no readily identifiable main activity bout and no clearly discernable onset of activity for any $24 \mathrm{hr}$ period (Fig. 1A). Periodogram analysis supported this contention, revealing multiple low-power periodic components $(20.7-28.7 \mathrm{hr}$; data not shown). In a minority of cases $(\sim 33 \%)$, a single apparent rhythmic component of wheel-running activity was discernable on visual inspection and subsequently confirmed by periodogram analysis (mean \pm SEM, $23.4 \pm 0.3 \mathrm{hr}$; range, 22.4-24.5) (Fig. $1 B$ ). This rhythm was typically in direct antiphase with the previous LD cycle.
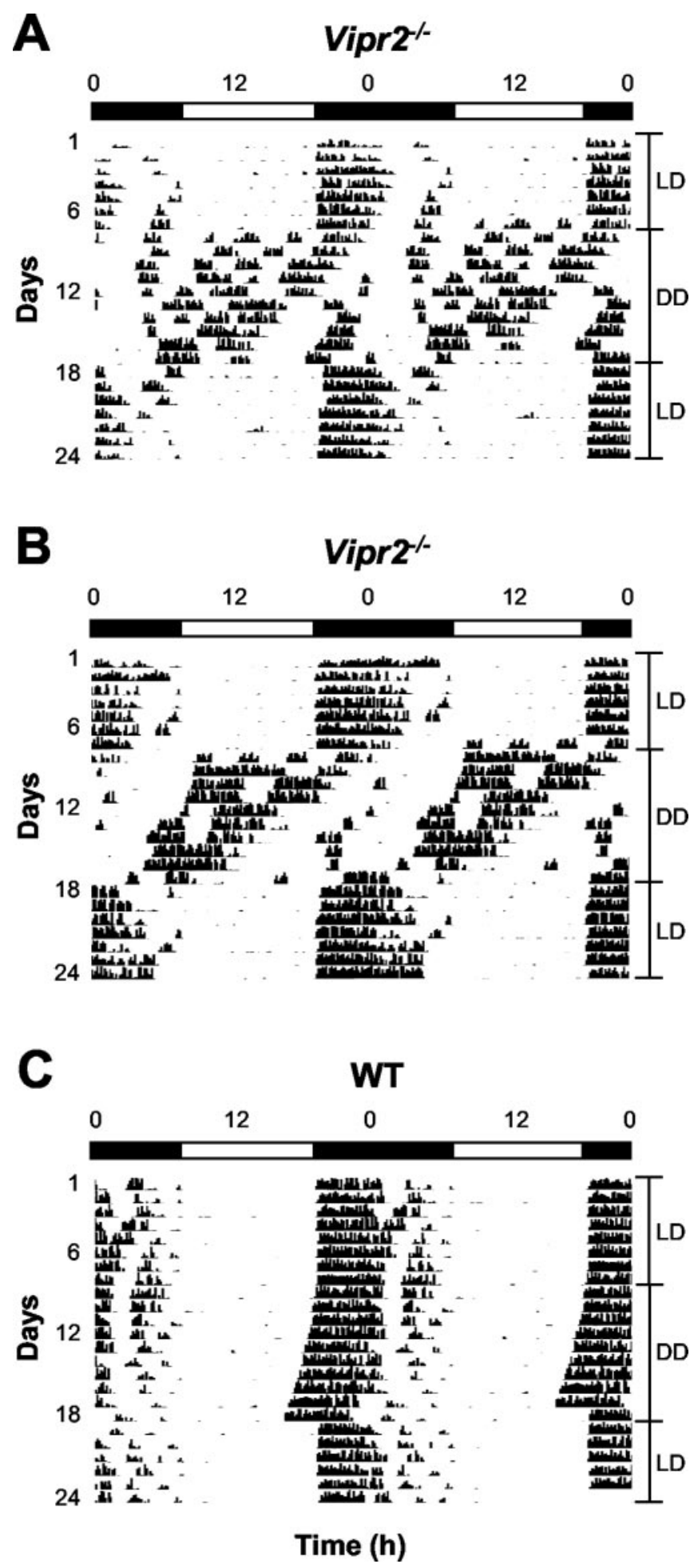

Figure 1. Wheel-running activity of mice in $L D$ and $D D . A, B, V i p r 2^{-1-}$ mice synchronize wheel-running activity to a $12 \mathrm{hr} L \mathrm{D}$ cycle and show severely disrupted temporal organization of locomotor activity in DD. $A$, The majority $(n=12$ of 18$)$ show no coherent circadian rhythmicity in wheel-running behavior in $\mathrm{DD}$. In this Vipr ${ }^{-1-}$ mouse, periodogram analysis revealed periodic components at 23.0, 24.4, 25.6 , and $29.1 \mathrm{hr}$. B, Some mice ( $n=6$ of 18) display a rhythmic component in locomotor activity with the main activity bout occurring in antiphase with the previous LD cycle. Periodogram analysis showed a clear $22.5 \mathrm{hr}$ rhythmic component in the activity of this Vipr2 ${ }^{-1-}$ mouse. C, WT mice entrain to a $12 \mathrm{hr}$ LD cycle and free-run in DD with a mean period of $23.8 \pm 0.1 \mathrm{hr}(n=8)$. Periodogram analysis showed a single clear periodic component of $23.7 \mathrm{hr}$ in this mouse. In $A-C$, activity is shown double plotted across 48 $\mathrm{hr}$. The light and dark bars represent lights on and off, respectively. 
All WT animals were entrained to the LD cycle. In DD, WT mice free-ran with a period of $23.8 \pm 0.1 \mathrm{hr}$ and maintained a distinct onset of activity per cycle $(n=8)$ (Fig. 1C), a behavioral phenotype similar to that reported previously (Schwartz and Zimmerman, 1990).

\section{Diurnal expression of pERK-ir in WT and Vipr2 ${ }^{-/-}$SCN}

Two-way ANOVA revealed a significant interaction between sampling time and genotype $\left(F_{(5,41)}=7.6 ; p<0.001\right)$. In the WT SCN under a LD cycle, pERK-ir showed significant variation over time with a late day peak (ZT10) and an early night nadir (ZT14) (Fig. 2 A). Pairwise comparisons in WT mice revealed that levels of pERK-ir at all day phases were significantly different from those at all night phases.

In contrast, pERK-ir levels in the SCN of Vipr $2^{-/-}$mice showed no significant temporal variation in expression; compared with peak expression in WT mice, levels were low across the entire diurnal cycle (Fig. 2A). Significant differences over time were only found at ZT10 versus ZT14, ZT18, and ZT22, with no statistically significant differences between any other time points.

\section{Circadian and photic regulation of pERK-ir}

Analysis of circadian expression and photic regulation using three-way ANOVA revealed that levels of pERK-ir in the SCN varied significantly over time, between genotypes, and with light treatment (light-pulsed vs non-lightpulsed animals; significant interaction between time $\times$ genotype $\times$ treatment; $\left.F_{(5,107)}=7.2 ; p<0.001\right)$ (Fig. 2 B, C). pERK-ir in the WT SCN in DD showed a distinct LD-like rhythmic circadian profile (Fig. 2 B). Pairwise comparisons revealed significant differences in levels of pERK-ir between all subjective day time points and all subjective night time points (except between CT2 and CT18). Subjective day light pulses failed to increase WT SCN pERK levels above those found in non-light-pulsed controls (i.e., than would be expected through endogenous circadian expression alone). However, light pulses given during the subjective night induced a significant increase in pERK-ir predominantly in the retinorecipient SCN (Fig. 2 B). Collectively, these data demonstrate phase gating of photic input to the SCN of WT mice.

In contrast, Vipr2 ${ }^{-/-}$SCN pERK expression was not rhythmic in DD (Fig. 2C); pairwise comparisons showed no significant differences between any time points, day or night. Photic induction of pERK in the Vipr2 $2^{-/-}$SCN also differed from WT animals. Light pulses presented at all subjective day and night time points increased pERK-ir levels compared with non-pulsed Vipr $2^{-/-}$controls (Fig. 2C). In all light-pulsed animals $(n=30)$, the level of photically induced pERK-ir was above the upper limit of the $95 \%$ CI for non-pulsed Vipr $2^{-/-}$control mice (Fig. 2D). Levels were elevated in all areas of the SCN. These data demonstrate a profound lack of phase gating of photic induction of pERK-ir in the SCN of Vipr $2^{-/-}$mice.
B

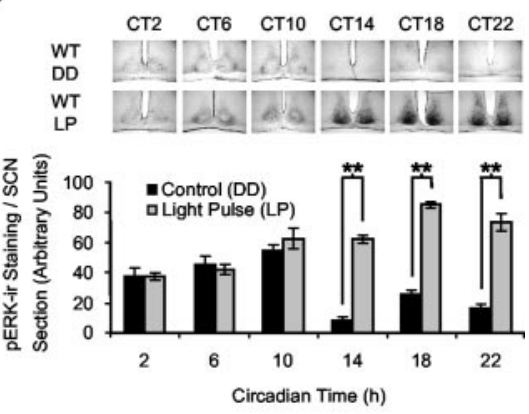

D

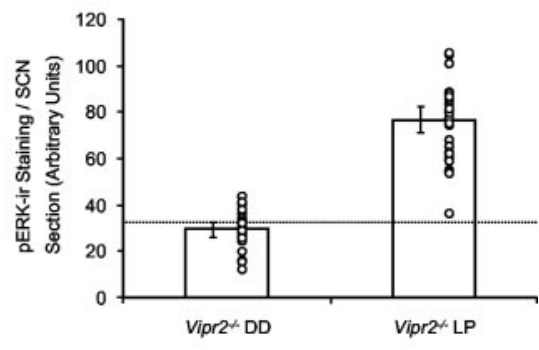

Figure 2. Diurnal, circadian, and gating of photic regulation of pERK in the SCN of WT mice but not Vipr2 ${ }^{-/-}$mice. A, Rhythmic expression of pERK-ir in the WT SCN; all day phases were significantly higher than all night phases (a priori comparisons, $p<0.005$ to $p<0.001)$. In the Vipr $2^{-1-} \mathrm{SCN}$, pERK-ir was constitutively low across the diurnal cycle. Significant differences were only ince subjective night but not during the subjective day $(p<0.001)$. C, pERK-ir in the Vipr2 ${ }^{-}$SCN did $(p<0.001)$. D, pERK-ir levels in all light-pulsed Vipr $2^{-/-}$mice were above the upper limit of the $95 \% \mathrm{Cl}$ (broken line) of non-pulsed Vipr $2^{-1-}$ mice, pooled from all circadian phases. The open circles depict data points from individual animals. Plots in $A-C$ depict the means \pm SEM, and histograms in D are the means $\pm 95 \% \mathrm{Cl}^{* *} p<0.001$. Scale bar, $50 \mu \mathrm{m}$.

\section{Expression of ERK-ir in WT and Vipr2 ${ }^{-/-}$SCN}

No rhythmic variation in total ERK protein levels was found for either genotype under LD or DD conditions, and ERK-ir levels were unchanged at all time points after a light pulse (data not shown).

\section{Diurnal expression of c-FOS-ir in WT and Vipr $2^{-/-} \mathrm{SCN}$}

The diurnal pattern of expression of c-FOS-ir was similar to that of pERK-ir in both WT and Vipr $2^{-1-}$ SCN, with a significant interaction of sampling time and genotype $\left(F_{(5,39)}=7.3 ; p<\right.$ 0.001). In WT mice, c-FOS expression at all day time points was significantly elevated versus all night time points. However, no significant differences were found between any day or night time points in the Vipr2 $2^{-/-}$SCN (Fig. 3A).

\section{Circadian and photic regulation of c-FOS-ir}

Circadian and photic regulation of c-FOS-ir resembled that of pERK. Three-way ANOVA revealed a significant effect of sampling time $\times$ genotype $\times$ light treatment on $\mathrm{c}$-FOS expression $\left(F_{(5,100)}=6.6 ; p<0.001\right)$ (Fig. $\left.3 B, C\right)$. In DD, the numbers of c-FOS-ir nuclei in the WT SCN were significantly higher during the mid-late subjective day (CT6, CT10) phase than during the subjective night and early subjective day phases. Subjective day light pulses failed to increase expression of c-FOS in WT mice, whereas subjective night light pulses induced significant increases in c-FOS-ir (Fig. 3B). In the Vipr $2^{-1-} \mathrm{SCN}$ in DD, there was no rhythmic expression in c-FOS-ir (no significant differences be- 
A

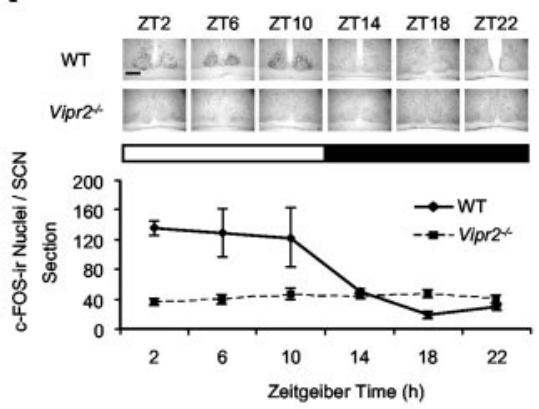

C

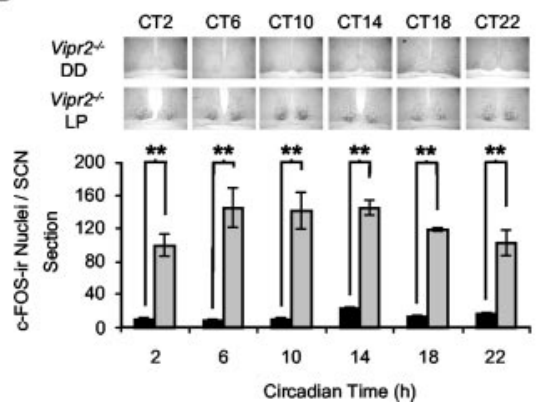

B

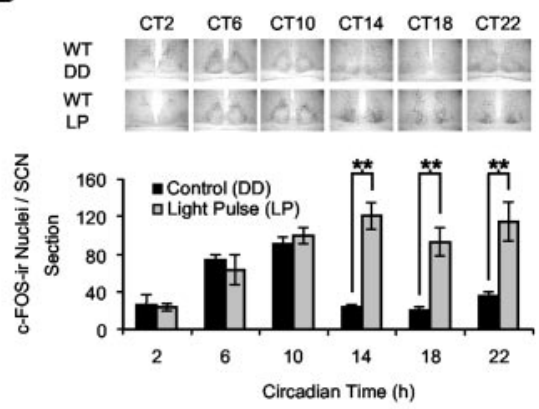

D

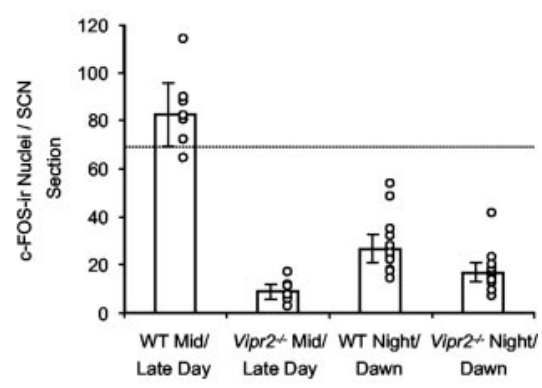

Figure 3. Diurnal, circadian, and gating of photic regulation of c-FOS in the SCN of WT mice but not Vipr2 ${ }^{-1-}$ mice. A, c-FOS-ir expression varied diurnally in the WT SCN; all day phases were significantly different from all night phases (a priori comparisons, $p<0.005$ to $p<0.001)$. In the Vipr2 ${ }^{-1-}$ SCN, c-FOS-ir did not vary across the diurnal cycle, with low numbers of c-FOS-ir nuclei at all time points (all comparisons, $p>0.05$ ). The light and dark bars represent lights on and off, respectively. $B, c-F 0 S$-ir in DD is rhythmically expressed across the circadian cycle in the WT SCN; mid and late subjective day phases were significantly different to all night and early day time points ( $p<0.05$ to 0.001). Light pulses (15 min) increased c-FOS-ir only during the subjective night in the WTSCN $(p<0.001)$. C, $c$-FOS-ir in the Vipr $2^{-1-}$ SCN did not vary over the circadian cycle (all comparisons, $p>0.05$ ). Light pulses increased numbers of c-FOS-ir nuclei at all circadian phases $(p<0.001)$. D, Spontaneous c-FOS-ir expression in the SCN of WT and Vipr2 ${ }^{-/-}$mice in DD at mid/late subjective day or subjective night/dawn. At all phases, levels of spontaneously expressed c-FOS-ir in the SCN of Vipr2 $2^{-1-}$ mice did not surpass the lower $95 \% \mathrm{Cl}$ (broken line) of peak c-FOS-ir expression during the $\mathrm{mid} /$ late subjective day in WT SCN. The open circles depict data points from individual animals. Plots in A-C depict the means \pm SEM, and histograms in $D$ are the means $\pm 95 \% \mathrm{Cl} .{ }^{* *} p<0.001$. Scale bar, $50 \mu \mathrm{m}$.

tween any time points). Consistent with the findings for pERK, light pulses significantly increased c-FOS-ir expression at all subjective day and night phases (Fig. $3 C$ ). Moreover, in all lightpulsed Vipr $2^{-1-}$ individuals, levels of c-FOS-ir were above the 95\% CI of c-FOS-ir expression in the SCN of non-pulsed Vipr $2^{-1-}$ controls (data not shown). At all phases, levels of spontaneously expressed c-FOS-ir in the SCN of Vipr $2^{-1-}$ mice did not surpass the lower CI of peak c-FOS-ir expression during the mid and late (CT6 and CT10 combined) day in WT SCN (Fig. 3D).

\section{Discussion}

Phase gating of sensitivity of the mammalian circadian system to light is a cardinal trait of a functional circadian oscillator. Here, we report for the first time the lack of phase gating of responses to photic stimulation in any mammalian model, demonstrating severe disruption to the circadian system of Vipr $2^{-/-}$mice. VIP has been proposed to be a key mediator of photic resetting of the circadian clock (Piggins et al., 1995; Reed et al., 2001). The circadian phenotypes of mice lacking the $\mathrm{VPAC}_{2}$ receptor $\left(\right.$ Vipr $^{-/-}$) (Harmar et al., 2002) or mice deficient in VIP (Colwell et al., 2003) demonstrate an additional, more fundamental role for VIP acting via the $\mathrm{VPAC}_{2}$ receptor in core clock rhythm generation and maintenance.

Levels of both pERK and c-FOS have previously been shown to oscillate in the mammalian SCN under the control of the circadian clock (Obrietan et al., 1998; Coogan and Piggins, 2003; Lee et al., 2003). Here, we show rhythmic expression of both of these factors in the WT mouse SCN under a LD cycle and in $\mathrm{DD}$, consistent with these previous findings. Under both lighting paradigms, pERK and c-FOS levels in the WT SCN varied significantly over the diurnal and circadian cycles, with high levels during the lights on/subjective day phase and low levels during lights off/subjective night phase. At lights on/ subjective day phases, pERK and c-FOS were predominantly localized to the shell region of the SCN, whereas at lights off/subjective night phases, neither pERK nor c-FOS showed distinct distributions.

In stark contrast, there was no obvious temporal variation in $\mathrm{pERK}$ or c-FOS expression in the Vipr2 $2^{-/-}$SCN in both LD and DD conditions. Indeed, there was no clear shell-core distinction in the distribution of pERK and c-FOS across the diurnal and circadian phases examined. The loss of rhythmic expression of these markers of cellular activation in the Vipr $2^{-/-} \mathrm{SCN}$ indicates functional deficits in the central circadian oscillator. These observations provide key support for the previous findings that rhythmic clock gene transcription, SCN neuronal firing rate, and behavioral activity are lacking in Vipr $2^{-/-}$ animals (Harmar et al., 2002; Cutler et al., 2003). It is noteworthy that disruption of ERK signaling ablates circadian variation in arginine vasopressin (AVP) mRNA transcription in rat SCN organotypic cultures (Arima et al., 2002) and that AVP mRNA expression in the Vipr $2^{-/-}$mouse is not rhythmic (Harmar et al., 2002). The demonstration that oscillations in $\mathrm{pERK}$ and c-FOS could not be driven by an environmental LD cycle provides additional evidence for a dysfunctional circadian clock. Arrhythmicity in pERK and c-FOS expression in LD (Figs. $2 A$, $3 A$ ) along with the induction of wheel running by daytime dark pulses (Harmar et al., 2002) suggests that the apparent entrainment of the Vipr2 $2^{-/-}$animals' locomotor behavior under LD can be explained by the direct suppression of activity by light (masking). Because it is now clear that the Vipr2 $2^{-/-} \mathrm{SCN}$ is responsive to light, it is intriguing that a nighttime light pulse, which substantially increased Per1-ir in the WT SCN, failed to alter expression of this core clock gene protein in the Vipr2 ${ }^{-/-}$SCN (Harmar et al., 2002).

The striking loss of phase gating of responses to photic input to the SCN in Vipr2 $2^{-1-}$ mice (Figs. $2 C, 3 C$ ) contrasts with the gated responses to light in the WT SCN, in which pERK and c-FOS were induced only during the subjective night. The mechanisms of phase gating in the SCN are poorly understood, although fluctuations in SCN NMDA receptor functionality are implicated (Pennartz et al., 2001). Moreover, NMDA receptor activation is crucial for the photic induction of pERK and c-FOS (Abe and Rusak, 1994; Coogan and Piggins, 2003).

In addition to the $\mathrm{VPAC}_{2}$ receptor, VIP binds with relatively low affinity to the $\mathrm{PAC}_{1}$ receptor, which is expressed in the SCN (Ajpru et al., 2002). The retinohypothalamic tract neurochemical, pituitary adenylate cyclase-activating polypeptide (PACAP) also binds to both $\mathrm{VPAC}_{2}$ and $\mathrm{PAC}_{1}$ receptors. However, transgenic mice lacking the $\mathrm{PAC}_{1}$ receptor or PACAP sustain circadian rhythms in light- 
entrainable locomotor activity rhythms (Hannibal et al., 2001; Kawaguchi et al., 2003). Collectively, these studies revealed that neither PACAP nor the $\mathrm{PAC}_{1}$ receptor is critical for maintenance of circadian rhythms or photic regulation of the SCN. This demonstrates that the altered circadian phenotype of Vipr $2^{-/-}$mice is not a generalized response to the absence of a peptide receptor but a specific effect arising from the loss of VIP-VPAC 2 signaling.

In DD, no Vipr2 $2^{-/-}$mice spontaneously expressed c-FOS-ir at peak levels seen in WT SCN (0 of 27) (Fig. 3D), and only a minority ( 6 of $18 ; \sim 33 \%$ ) of these mice showed apparent circadian rhythmicity in wheel-running behavior. However, subsequent to light-pulse treatment during subjective day or night, all Vipr $2^{-1-}$ individuals $(n=55)$ expressed pERK-ir or c-FOS-ir at levels above the $95 \%$ CI of non-pulsed Vipr $2^{-/-}$mice. This suggests that in the absence of VIP-VPAC ${ }_{2}$ signaling, oscillatory and gating mechanisms within the SCN have become decoupled.

These results lend partial support to the idea that the SCN consists of rhythmic oscillator cells and non-rhythmic "gate" cells (Antle et al., 2003). Accordingly, retinally innervated VIP cells in the ven$\mathrm{tral} /$ core SCN may act as the gate cells, whereas cells that receive VIP input and express $\mathrm{VPAC}_{2}$ receptors may function as oscillator cells. AVP-containing cells of the dorsomedial/shell SCN express $\mathrm{VPAC}_{2}$ mRNA (Kallo et al., 2002) and receive inputs from VIP neurons (Romijn et al., 1997; Abrahamson and Moore, 2001). Exogenous VIP resets the rhythm of release of AVP from rat SCN organotypic cultures in vitro (Watanabe et al., 2000). AVP cells also reciprocally innervate some of these VIP cells (Romijn et al., 1997). Therefore, in the absence of VIP-VPAC 2 signaling, such as in the Vipr $2^{-/-}$mouse SCN, the VIP gate cells no longer communicate effectively with the oscillator AVP neurons, resulting in these autonomous cellular oscillators drifting apart and defaulting to an apparent arrhythmic state. Consequently, circadian information is not conveyed to VIP cells, and these gate cells lose temporal variation in responsiveness to sensory cues.

Concordant with this interpretation, an in vitro study of a per1-driven luciferase reporter in rat (Yamaguchi et al., 2003) established that blockade of synaptic transmission decouples rhythmic clock gene expression in the SCN and abolishes high amplitude rhythms. Our previous observations that there are very few high firing neurons and no detectable circadian variation in electrical activity in the Vipr ${ }^{-1-}$ mouse SCN in vitro (Cutler et al., 2003) support a general model that cell-cell communication is key for sustaining normal circadian function. The apparent robust rhythmicity in wheel running in a minority of Vipr $2^{-1-}$ individuals suggests either that low-amplitude oscillations of nuclear and cellular activity in the SCN are sufficient to drive some aspects of behavioral rhythmicity or that extra-SCN oscillators may govern the temporal organization of locomotor activity.

Therefore, our data, taken in context with that of Harmar et al. (2002) and Cutler et al. (2003), suggest that the VIP-VPAC sig- $^{2}$ naling system performs a critical function in circadian rhythm generation and maintenance. Furthermore, in the absence of VIP-VPAC 2 signaling, the murine SCN remains sensitive to light and indeed appears to be permanently locked in a photosensitive phase. This provides the first demonstration of disrupted phase gating to a Zeitgeber in any mammalian model.

\section{References}

Abe H, Rusak B (1994) Physiological mechanisms regulating photic induction of Fos-like protein in hamster suprachiasmatic nucleus. Neurosci Biobehav Rev 18:531-536.
Abrahamson EE, Moore RY (2001) Suprachiasmatic nucleus in the mouse: retinal innervation, intrinsic organization, and efferent projections. Brain Res 916:172-191.

Ajpru S, McArthur AJ, Piggins HD, Sugden D (2002) Identification of PAC receptor isoform mRNAs by real-time PCR in rat suprachiasmatic nucleus. Mol Brain Res 105:29-37.

Allada R, Emery P, Takahashi JS, Rosbash M (2001) Stopping time: the genetics of fly and mouse circadian clocks. Annu Rev Neurosci 24:1091-1119.

Antle MC, Foley DK, Foley NC, Silver R (2003) Gates and oscillators: a network model of the brain clock. J Biol Rhythms 18:339-350.

Arima H, House SB, Gainer H, Aguilera G (2002) Neuronal activity is required for the circadian rhythm of vasopressin gene transcription in the suprachiasmatic nucleus in vitro. Endocrinology 143:4165-4171.

Colwell CS, Michel S, Itri J, Rodriguez W, Tam J, Lelievre V, Hu Z, Liu X, Waschek JA (2003) Disrupted circadian rhythms in VIP- and PHIdeficient mice. Am J Physiol 285:R939-R949.

Coogan AN, Piggins HD (2003) Circadian and photic regulation of phosphorylation of ERK1/2 and Elk-1 in the suprachiasmatic nuclei of the Syrian hamster. J Neurosci 23:3085-3093.

Cutler DJ, Haraura M, Reed HE, Shen S, Sheward WJ, Morrison CF, Marston HM, Harmar AJ, Piggins HD (2003) The mouse $\mathrm{VPAC}_{2}$ receptor confers suprachiasmatic nuclei cellular rhythmicity and responsiveness to vasoactive intestinal polypeptide in vitro. Eur J Neurosci 17:197-204.

Gillette MU, Mitchell JW (2002) Signaling in the suprachiasmatic nucleus: selectively responsive and integrative. Cell Tissue Res 309:99-107.

Hannibal J, Jamen F, Nielsen HS, Journot L, Brabet P, Fahrenkrug J (2001) Dissociation between light-induced phase shift of the circadian rhythm and clock gene expression in mice lacking the pituitary adenylate cyclaseactivating polypeptide type 1 receptor. J Neurosci 21:4883-4890.

Harmar AJ, Marston HM, Shen S, Spratt C, West KM, Sheward WJ, Morrison CF, Dorin JR, Piggins HD, Reubi J-C, Kelly JS, Maywood ES, Hastings $\mathrm{MH}$ (2002) The VPAC 2 receptor is essential for circadian function in the mouse suprachiasmatic nuclei. Cell 109:497-508.

Kallo I, Kalamatianos T, Wiltshire N, Shen S, Sheward JW, Harmar AJ, Coen CW (2002) Transgenic approach cell-specific expression of the VPAC2 receptor in the mouse suprachiasmatic nucleus. Soc Neurosci Abstr 28:474.11.

Kawaguchi C, Tanaka K, Isojima Y, Shintani N, Hashimoto H, Baba A, Nagai K (2003) Changes in light-induced phase shift of circadian rhythm in mice lacking PACAP. Biochem Biophys Res Commun 310:169-175.

Lee HS, Nelms JL, Nguyen M, Silver R, Lehman MN (2003) The eye is necessary for a circadian rhythm in the suprachiasmatic nucleus. Nat Neurosci 6:111-112.

Obrietan K, Impey S, Storm DR (1998) Light and circadian rhythmicity regulate MAP kinase activation in the suprachiasmatic nuclei. Nat Neurosci 8:693-700.

Pennartz CM, Hamstra R, Geurtsen AM (2001) Enhanced NMDA receptor activity in retinal inputs to the rat suprachiasmatic nucleus during the subjective night. J Physiol (Lond) 532:181-194.

Piggins HD, Cutler DJ (2003) The roles of vasoactive intestinal polypeptide in the mammalian circadian clock. J Endocrinol 177:7-15.

Piggins HD, Antle MC, Rusak B (1995) Neuropeptides phase shift the mammalian circadian pacemaker. J Neurosci 15:5612-5622.

Reed HE, Meyer-Spasche A, Cutler DJ, Coen CW, Piggins HD (2001) Vasoactive intestinal polypeptide (VIP) phase-shifts the rat suprachiasmatic nucleus clock in vitro. Eur J Neurosci 13:839-843.

Reppert SM, Weaver DR (2002) Coordination of circadian timing in mammals. Nature 418:935-941.

Romijn HJ, Sluiter AA, Pool CW, Wortel J, Buijs RM (1997) Evidence from confocal fluorescence microscopy for a dense reciprocal innervation between AVP-, Som-, VIP/PHI-, GRP-, and VIP/PHI/GRP-immunoreactive neurons in the rat suprachiasmatic nucleus. Eur J Neurosci 9:2613-2623.

Rusak B, Zucker I (1979) Neural regulation of circadian rhythms. Physiol Rev 59:449-526.

Schwartz WJ, Zimmerman P (1990) Circadian timekeeping in BALB/c and C57BL/6 inbred mouse strains. J Neurosci 11:3685-3694.

Watanabe K, Vanecek J, Yamaoka S (2000) In vitro entrainment of the circadian rhythm of vasopressin-releasing cells in suprachiasmatic nucleus by vasoactive intestinal polypeptide. Brain Res 877:361-366.

Yamaguchi S, Isejima H, Matsuo T, Okura R, Yagita K, Kobayashi M, Okamura H (2003) Synchronization of cellular clocks in the suprachiasmatic nucleus. Science 302:1408-1412. 